\title{
1 Better model transfers require knowledge of mechanisms
}

2 Phil J. Bouchet ${ }^{1, *}$, A. Townsend Peterson ${ }^{2}$, Damaris Zurell ${ }^{3,4}$, Carsten F. Dormann ${ }^{5}$, David Schoeman ${ }^{6,7}$, 3 Rebecca E. Ross ${ }^{8,9}$, Paul Snelgrove ${ }^{10}$, Ana M.M. Sequeira ${ }^{11,12}$, Mark J. Whittingham ${ }^{13}$, Lifei Wang ${ }^{14,15}$, 4 Giovanni Rapacciuolo ${ }^{16}$, Steffen Oppe ${ }^{17}$, Camille Mellin ${ }^{18,19}$, Valentina Lauria ${ }^{20}$, Periyadan K. 5 Krishnakumar ${ }^{21}$, Alice R. Jones ${ }^{18}$, Stefan Heinänen ${ }^{22,23}$, Risto K. Heikkinen ${ }^{24}$, Edward J. Gregr ${ }^{25,26}$, Alan 6 H. Fielding ${ }^{27}$, M. Julian Caley ${ }^{28,29}$, A. Márcia Barbosa ${ }^{30}$, Andrew J. Bamford ${ }^{31}$, Hector Lozano-Montes ${ }^{32}$, 7 Stephen Parnell ${ }^{33}$, Seth Wenger ${ }^{34}$, Katherine L. Yates ${ }^{33}$

\footnotetext{
${ }^{1}$ Centre for Research into Ecological \& Environmental Modelling, School of Mathematics and Statistics, University of St Andrews, St Andrews, UK

2 Biodiversity Institute, University of Kansas, Lawrence, KS 66045, USA

${ }^{3}$ Swiss Federal Research Institute WSL, Dept. Landscape Dynamics, Zuercherstrasse 111, CH-8903 Birmensdorf, Switzerland

${ }^{4}$ Humboldt-Universität zu Berlin, Geography Dept., Unter den Linden 6, D-10099 Berlin, Germany

${ }^{5}$ Biometry \& Environmental System Analysis, University of Freiburg, Tennenbacher Str. 4, 79106 Freiburg, Germany

${ }^{6}$ School of Science \& Engineering, The University of the Sunshine Coast, Maroochydore, QLD 4558, Australia

${ }^{7}$ Centre for African Conservation Ecology, Department of Zoology, Nelson Mandela University, Port Elizabeth, South Africa

${ }^{8}$ School of Biological and Marine Sciences, Plymouth University, Drake Circus, Plymouth, PL4 8AA, UK

9 Institute for Marine Research, Nordnesgaten 50, 5005 Bergen, Norway

${ }^{10}$ Department of Ocean Sciences and Department of Biology, Memorial University of Newfoundland, St. John's, NL A1C 5S7, Canada

${ }^{11}$ School of Biological Sciences, University of Western Australia, 35 Stirling Highway, Crawley, WA 6009, Australia

12 IOMRC and The University of Western Australia Oceans Institute, University of Western Australia, Crawley, WA 6009, Australia

${ }^{13}$ Biology, School of Natural and Environmental Sciences, Newcastle University, Newcastle-Upon-Tyne, NE1 7RU, UK

${ }^{14}$ Department of Ecology and Evolutionary Biology, University of Toronto, Toronto, ON M5S 3B2, Canada

${ }^{15}$ Gulf of Maine Research Institute, Portland, ME 04101, USA

${ }^{16}$ University of California, Merced, 5200 N Lake Rd, Merced, CA 95230, USA

${ }^{17}$ RSPB Centre for Conservation Science, Royal Society for the Protection of Birds, The David Attenborough Building, Pembroke Street, Cambridge CB2 3QZ, UK

18 The Environment Institute and School of Biological Sciences, University of Adelaide, Adelaide, SA 5005, Australia

${ }^{19}$ Australian Institute of Marine Science, PMB No 3, Townsville 4810, QLD, Australia

20 Instituto per l'Ambiente Marino Costiero, IAMC-CNR, Mazara del Vallo, Trapani, Italy

${ }^{21}$ Center for Environment and Water, Research Institute, King Fahd University of Petroleum and Minerals, Dhahran 31261, Saudi Arabia

22 DHI, Ecology and Environment Department, Agern Allé 5, DK-2970 Hørsholm, Denmark

${ }^{23}$ Novia University of Applied Sciences, Raseborgsvägen 9, 10600 Ekenäs, Finland

${ }^{24}$ Finnish Environment Institute, Biodiversity Centre, PO Box 140, FIN- 00251 Helsinki, Finland

25 Institute for Resources, Environment, and Sustainability, University of British Columbia, AERL Building, 2202 Main Mall Vancouver, BC, Canada

${ }^{26}$ SciTech Environmental Consulting, 2136 Napier Street, Vancouver, BC V5L 2N9, Canada

${ }^{27}$ Haworth Conservation Ltd, Bunessan, Isle of Mull, Scotland

${ }^{28}$ ARC Centre for Excellence in Mathematical and Statistical Frontiers, Queensland University of Technology, Brisbane, QLD, Australia

${ }^{29}$ School of Mathematical Sciences, Queensland University of Technology, Brisbane, QLD, Australia

${ }^{30}$ Centro de Investigação em Ciências Geo-Espaciais, Faculdade de Ciências, Universidade do Porto, Observatório Astronómico Prof. Manuel de Barros, Alameda do Monte da Virgem, 4430-146 Vila Nova de Gaia, Portugal

31 Wildfowl \& Wetlands Trust, Slimbridge, Gloucestershire, GL2 7BT, UK

32 CSIRO Oceans \& Atmosphere, Indian Ocean Marine Research Centre, The University of Western Australia, Crawley, WA 6009, Australia

33 School of Environment and Life Sciences, University of Salford, Manchester, UK

34 Odum School of Ecology, University of Georgia, Athens, GA 30601, USA
} 
Model transferability is an emerging and important branch of predictive science that has grown primarily from a need to produce ecological forecasts in the face of widespread data deficiency and escalating environmental novelty. In our recent article in TREE [1], we outlined some of the major roadblocks that currently undermine the practice of model transfers in ecology. Radchuk et al. [2]'s response to our work stresses the value of considering 'first principles' in projections of ecosystem change [3], and offers insights into outstanding challenges specific to mechanistic (synonym: process-based) models [4].

We strongly agree that improving ecological prediction under novel conditions requires a mechanistic understanding of natural systems [5]. Indeed, several of the research priorities we identified reflect this very idea (see [1] - Box 3 and pp. 795, 799). However, as Radchuk et al. [2] point out, the majority of mechanistic models are data-hungry by nature and rely heavily on imposed parameters derived from field observations or empirical relationships [6]. Given the real-world constraints of data availability, obtaining the detailed measurements necessary for robust model calibration and setup is not only time-consuming but also costly [6], such that mechanistic models have only been successfully built for the most charismatic, well-studied, and/or economically valuable species [7]. This limits their utility to support many of the management decisions that model transfers could inform. Data constraints also mean that Radchuk et al. [2]'s recommendation to capture what are largely unquantified and dynamic biotic interactions (e.g. competition, facilitation, predation) appears, for now, more aspirational than realistic. Borrowing information from related (and better known) taxa can partially circumvent the problem of data scarcity [7], but often at the cost of accepting unverified assumptions about parameter validity, and with potentially large biases in model outputs introduced by seemingly trivial changes in parameter values and initial conditions [6]. This uncertain behaviour perhaps explains why process-based models have received less attention in the literature to date, and remain less prominent overall in the context of model transfers [1].

While we see tremendous appeal in a process-based view of ecological inquiry, we therefore wish to temper general expectations. Significant advances in data collection are still imperative to pushing the discipline forward [8], and model transfers remain most urgently needed in knowledge-poor contexts [1], where information gaps make correlative descriptions of patterns the only viable pathway to ecological prediction. As a result, ecologists have proven rather slow to embrace mechanistic approaches [9]. For instance, although dynamic vegetation models built on first principles (e.g. physiology, photosynthesis) have been available for a few decades, they are either only applicable at coarse spatial resolutions or need detailed parameterisations to local site conditions [9]. Likewise, animal ecology has only very recently started to consider first principles such as dynamic energy budgets or foraging theory for modelling population dynamics reflecting individual-based processes [9].

Importantly, and as Radchuk et al. [2] remind us, mechanistic and correlative models also share many of the same underlying issues (e.g. equifinality, nonstationarity, model misspecification, model complexity) [4]. Rigorous tests of mechanistic models in non-analogue contexts are largely lacking (but see $[10,11]$ ), meaning that external model evaluation should be seen as a critical step in determining their benefits for transferability. Until this is addressed, the relative value of mechanistic models over correlative models will arguably remain equivocal [11], and neither 'correlationists' nor 'mechanists' should thus feel entitled to claim holding the moral high ground [4]. 
79 80

Ultimately, the complexity of conservation challenges in the Anthropocene requires that we invest in finding efficient solutions grounded in an understanding of the inner workings of nature [3]. A modelling philosophy that allows parameters to naturally emerge from first principles could offer exciting opportunities to attain this goal, as long as it is subjected to meticulous testing and that principles can be defined explicitly and consistently $[7,12]$. Without a common and consistent definition, one ecologist's first principles could easily become another's phenomenologies [12], making transfers strongly dependent on correctly identifying the key processes driving system behaviour in the first place, lest the model fails [7]. Whilst we agree with Radchuk et al. [2] that both mechanistic and correlative models are equally valuable, the latter still remain, in many cases, the most utilisable. As a result, we argue that the most immediate advances in transferability will be achieved by encouraging the development of correlative models grounded in well-established mechanisms [1].

\section{References}

[1] Yates, K.L. et al. (2018) Outstanding challenges in the transferability of ecological models. Trends Ecol. Evol. 33, 790-802

[2] Radchuk, V. et al. (2019) Transferability of mechanistic ecological models is about emergence. Trends Ecol. Evol. DOI: 10.1016/j.tree.2019.01.010

[3] Marquet, P.A. et al. (2015) On the importance of first principles in ecological theory development. BioScience 65, 342-343

[4] Dormann, C.F. et al. (2012) Correlation and process in species distribution models: Bridging a dichotomy. J. Biogeogr. 39, 2119-2131

[5] Cuddington, K. et al. (2013) Process-based models are required to manage ecological systems in a changing world. Ecosphere 4, art20

[6] Peterson, A.T. et al. (2015) Mechanistic and correlative models of ecological niches. Eur. J. Ecol. 1, 28-38

[7] Evans, M.E.K. et al. (2016) Towards process-based range modeling of many species. Trends Ecol. Evol. 31, 860-871

[8] Schurr, F.M. et al. (2012) How to understand species' niches and range dynamics: A demographic research agenda for biogeography. J. Biogeogr. 39, 2146-2162

[9] Grimm, V. and Berger, U. (2016) Structural realism, emergence, and predictions in next-generation ecological modelling: Synthesis from a special issue. Ecol. Modell. 326, $177-187$

[10] Fordham, D.A. et al. (2018) How complex should models be? Comparing correlative and mechanistic range dynamics models. Glob. Chang. Biol. 24, 1357-1370

[11] Shabani, F. et al. (2016) A comparison of absolute performance of different correlative and mechanistic species distribution models in an independent area. Ecol. Evol. $6,5973-5986$

[12] Houlahan, J.E. et al. (2015) On theory in ecology: Another perspective. BioScience $65,341-342$ 